'Unidad Docente Asistencial en Enfermedades Autoinmunes Sistémicas y Reumatológicas. Clínica Médica, Departamento de Medicina, Hospital Pasteur, Administración de Servicios de Salud del Estado (ASSE). Facultad de Medicina, Universidad de la República. Montevideo, Uruguay.

${ }^{2}$ Unidad de Investigación de Enfermedades Autoinmunes. Servicio de Medicina Interna. BioCruces Health Research Institute. Hospital Universitario

Cruces. Universidad del País Vasco (UPV)/Euskal Herriko Unibertsitatea (EHU). Barakaldo, España.

Recibido el 19 de mayo de 2015, aceptado el 22 de septiembre de 2015.

Correspondencia a: Dr. Álvaro Danza. Almirón 5082, CP 11400. Montevideo, Uruguay. alvarodanza@gmail.com

\section{Hidroxicloroquina en el tratamiento de las enfermedades autoinmunes sistémicas}

\author{
ÁLVARO DANZA ${ }^{1,2}$, DIEGO GRAÑA ${ }^{1}$, MABEL GOÑI ${ }^{1}$, \\ ANDREA VARGAS ${ }^{1}$, GUILLERMO RUIZ-IRASTORZA ${ }^{2}$
}

\section{Hydroxychloroquine for autoimmune diseases}

Hydroxychloroquine (HCQ) is by far the most frequently used antimalarial for the management of Systemic Autoimmune Diseases. It has immunomodulatory, hypolipidemic, hypoglycemic and antithrombotic properties and it diminishes the risk of malignancies. The most important mechanisms to explain the immunomodulatory actions are its ability to reduce inflammatory pathways and Toll-like receptors activation. The safety profile is favorable. In spite of its low frequency, retinal toxicity is potentially severe. In systemic lupus erythematous HCQ therapy reduces activity, the accrual of organ damage, risk of infections and thrombosis and improves the cardiometabolic profile. It contributes to induce lupus nephritis remission, spares steroid use and increases survival rates. In rheumatoid arthritis, it improves cardiometabolic risk and has a favorable effect in joint inflammation. In Sjögren's syndrome, an increased lacrimal quality as well as an improvement in objective and subjective inflammatory markers has been demonstrated with HCQ. In Antiphospholipid Syndrome, HCQ is effective in primary and secondary thrombosis prevention. The effectiveness of the drug in other systemic autoimmune diseases is less established. HCQ therapy may improve dermatological manifestations in Dermatomyositis and may have a positive effects in the treatment of Sarcoidosis and Still disease.

(Rev Med Chile 2016; 144: 232-240)

Key words: Antiphospohlipid syndrome; Autoimmune diseases; Hydroxychloroquine, systemic lupus erythematosus; Rheumatoid arthritis; Sjögren's syndrome.
L as enfermedades autoinmunes sistémicas (EAS) constituyen un conjunto de entidades caracterizadas por la presencia de autoanticuerpos dirigidos contra diversos parénquimas, lo que determina manifestaciones clínicas variables y frecuentemente difíciles de sistematizar. Eventualmente cualquier parénquima puede expresar manifestaciones de la enfermedad. Son característicos el compromiso cutáneo, mucoso, articular, seroso, de las glándulas salivales, hematológico, nefrológico, oftalmológico y del sistema nervioso central, entre otros ${ }^{1}$.

El tratamiento de las EAS clásicamente com- prende diversos fármacos, entre los que se destacan glucocorticoides, antiinflamatorios no esteroideos, inmunosupresores, antimaláricos y, más recientemente, terapias biológicas dirigidas contra blancos moleculares específicos ${ }^{2,3}$.

Los antimaláricos han sido ampliamente empleados en el tratamiento de las EAS, especialmente en lupus eritematoso sistémico (LES) y artritis reumatoide (AR), desde hace largo tiempo. El primero en emplearse fue quinina, hace ya casi 100 años, para el tratamiento del LES. Actualmente se emplean hidroxicloroquina (HCQ), cloroquina (CQ) y quinacrina ${ }^{4}$. 
Hidroxicloroquina es, por lejos, la más utilizada en el tratamiento de las EAS y otras enfermedades reumatológicas. Sin embargo, no en todos los casos en que se la emplea están claros los beneficios que su uso determina.

El objetivo de esta revisión es actualizar los aspectos farmacológicos más relevantes, los beneficios del tratamiento con HCQ en diversas enfermedades autoinmunes y reumatológicas y la seguridad de su uso, proponiendo en qué casos el tratamiento es más adecuado.

\section{Hidroxicloroquina-farmacología clínica}

Los antimaláricos tienen algunas particularidades farmacológicas relevantes. Es característica la variabilidad interindividual en la absorción, además, las concentraciones plasmáticas pueden alterarse con el nivel de actividad inflamatoria de la enfermedad, por lo que también existe variabilidad intraindividual. Se distribuyen ampliamente en el tejido muscular, hígado, bazo, riñones, pulmones, células de la sangre, glándulas pituitaria y suprarrenal y tejidos que contienen melanina. Se metabolizan en el hígado y se excretan por vía renal. La vida media de eliminación de HCQ es de 30 a 50 días, el tiempo que demora en comenzar a hacer su efecto antiinflamatorio es de 4 a 6 semanas, mientras que el tiempo en llegar a concentraciones estables en sangre se estima entre 4 y 6 meses $^{5}$. Se recomienda que la dosis de uso no supere los $6,5 \mathrm{mg} / \mathrm{kg} / \mathrm{dí}$ a, tomando como referencia el peso ideal del paciente ${ }^{6}$.

A nivel hepático se ha planteado una posible interacción con el tabaquismo. Es bien conocido que el tabaco es inductor del CYP450, lo que sugiere que puede disminuir el efecto de la HCQ. Existen estudios que confirman una disminución del efecto de la HCQ sobre las lesiones cutáneas del LES en fumadores ${ }^{7}$. Sin embargo, recientemente estas observaciones han sido puestas en duda, lo que abre un terreno de estudio e investigación ${ }^{8}$.

El mecanismo por el cual HCQ ejerce su efecto inmunomodulador es complejo y no completamente aclarado. Se acepa que controla el proceso de autoinmunidad por varias vías, dentro de lo que se destaca su capacidad por interferir con la presentación de autoantígenos, bloquear la respuesta de linfocitos T inducida por antígenos, disminuir la producción de mediadores inflamatorios e inhibir la activación de los receptores Toll-like (TLR) ${ }^{9}$.
Los antimaláricos interfieren con la función de fagocitosis, lo que determina una alteración en la presentación de antígenos, fundamentalmente autoantígenos y, en menor medida, de antígenos exógenos. En el mismo sentido, bloquean la proliferación de linfocitos $\mathrm{T}$, al tiempo que determinan una disminución en la producción de citoquinas involucradas en la respuesta inflamatoria, IL-1, IL-2, IL-6, IL-17, IL-22, interferón $\alpha, \gamma$ y factor de necrosis tumoral $\alpha$. Sin embargo, el mecanismo de acción probablemente más importante está relacionado con la inhibición de la activación de algunos TLR, específicamente los intracelulares que reconocen ácidos nucleicos (TLR 3, 7, 8 y 9). Estos receptores están involucrados en la inmunidad innata y tienen un rol clave en las EAS. Se ha establecido que los TLR se unen a los ácidos nucleicos, promoviendo la producción de autoanticuerpos y mediadores inflamatorios, con el consecuente daño tisular. Esta interacción requiere de un $\mathrm{pH}$ ácido a nivel de los lisosomas. Los antimaláricos, que son bases débiles, inhiben la acidificación del lisosoma y, en consecuencia, son capaces de inhibir la interacción entre los TLR y los ácidos nucleicos. Esto determina, por ejemplo, en modelos experimentales de AR, una disminución en la producción de factor reumatoide. En experimentos básicos también se ha verificado que este efecto provoca disminución de otras moléculas inflamatorias involucradas en el daño tisular de la AR, el LES y otras EAS ${ }^{9-11}$.

En los últimos tiempos se ha sugerido que la dosificación de los niveles plasmáticos de HCQ podría ser de utilidad en la monitorización del tratamiento. Algunos estudios han sugerido que niveles plasmáticos por encima de $1.000 \mathrm{ng} / \mathrm{ml}$ coinciden con menor actividad del LES y menos frecuencia de brotes. Sin embargo, esto no se ha confirmado consistentemente. La variabilidad intra e interindividual y la accesibilidad a la dosificación de niveles plasmáticos de HCQ dificulta la utilidad de esta técnica. Por el momento está establecido que su mayor utilidad es para verificar la adhesión al tratamiento9.

La seguridad del tratamiento con HCQ ha sido largamente analizada. Los efectos adversos más frecuentes son leves, incluyendo molestias gastrointestinales, prurito acuógeno y pigmentación cutánea. Suelen aparecer al inicio y mejorar con el mantenimiento del tratamiento, excepcionalmente determinan su interrupción ${ }^{12}$. Si bien HCQ 
no provoca psoriasis, se ha propuesto que su uso puede determinar exacerbación de la enfermedad, lo cual no se ha confirmado consistentemente ${ }^{13}$.

El efecto adverso más temido es su potencial daño oftalmológico, específicamente a nivel de la retina. El mecanismo por el cual estos medicamentos pueden dañar la retina no está claramente establecido, pero es conocido que los antimaláricos se unen a la melanina de la capa epitelial pigmentada de la retina, pudiendo afectar a los conos y bastones. Existen lesiones precoces, denominadas pre-maculares. Se manifiestan como edema, granularidad y pigmentación macular. Se puede detectar mediante la objetivación de un escotoma paracentral al color rojo en el test de colores. Se trata de una lesión potencialmente reversible que, en caso de detectarse y suspender el tratamiento en ese momento permitiría evitar la progresión al extremo más temido e irreversible, la maculopatía en "ojo de buey". Esta última se puede manifestar con escasos síntomas o bien con escotomas, nictalopía (dificultad para ver con escasa luz) y pérdida de la agudeza visual ${ }^{14}$. La frecuencia de estas complicaciones con el tratamiento con HCQ es muy baja, en el orden de 1 a 3/1.000 pacientes expuestos durante los primeros 5 a 10 años de tratamiento ${ }^{15}$. Se consideran factores de riesgo para su aparición: el tratamiento con dosis acumuladas superiores a 1.000 gramos; las dosis diarias mayores a $6,5 \mathrm{mg} /$ kg de peso ideal; la edad avanzada; la enfermedad renal o hepática y la presencia de enfermedad retiniana o maculopatía previa. En consecuencia, el tratamiento con HCQ requiere control oftalmológico. La Academia Americana de Oftalmología ha pautado un control al inicio del tratamiento y anual luego de 5 años de tratamiento, siempre que el paciente esté asintomático o anual en caso de tratarse de pacientes con alguno de los factores de riesgo antes mencionados ${ }^{16}$.

A nivel cardiovascular se han reportado trastornos de la conducción y falla cardiaca por antimaláricos, pero ambas complicaciones son muy poco frecuentes con el uso de HCQ. Puede manifestarse como bloqueos de la conducción aurículo ventricular o bloqueos de rama, mientras que el compromiso miocárdico se presenta con un patrón restrictivo e hipertrofia biventricular. $\mathrm{Su}$ confirmación requiere biopsia miocárdica, aunque puede optarse por biopsia muscular, puesto que las manifestaciones anatomopatológicas a nivel del músculo estriado son coincidentes. La discon- tinuación del tratamiento puede determinar una lenta mejoría de estas complicaciones. No está establecido que se requieran controles específicos para detectar precozmente estas complicaciones ${ }^{17}$.

Por último, cabe destacar que el fármaco es seguro durante el embarazo y lactancia. Su interrupción es riesgosa para la gestación, por lo que debe mantenerse durante todo el período grávido-puerperal ${ }^{18,19}$.

\section{Hidroxicloroquina en el lupus eritematoso sistémico}

Hidroxicloroquina se emplea desde hace años en el tratamiento del $\mathrm{LES}^{20}$.

Probablemente es en esta enfermedad donde más se ha avanzado en relación al conocimiento sobre sus beneficios terapéuticos. Su eficacia y seguridad ha sido ampliamente comprobada en varios estudios clínicos y sistematizada en diversas publicaciones ${ }^{9,15}$. Los beneficios más relevantes se resumen en la Tabla 1. En la Tabla 2 se sintetizan los beneficios del tratamiento con HCQ en diversas EAS.

Tabla 1. Efectos del tratamiento con hidroxicloroquina en el lupus eritematoso sistémico

\begin{tabular}{|ll|}
\hline Sobrevida & Aumento \\
\hline Infecciones & Disminución del riesgo \\
\hline Actividad & $\begin{array}{l}\text { Disminución frecuencia } \\
\text { y severidad de brotes }\end{array}$ \\
\hline Trombosis & Disminución \\
\hline Daño orgánico acumulado & Disminución \\
\hline Riesgo cardiometabólico & Mejoría \\
\hline Riesgo de desarrollar neoplasias & Disminución \\
\hline Consumo de glucocorticoides & Disminución \\
\hline
\end{tabular}

Tabla 2. Beneficios del uso de hidroxicloroquina en diversas enfermedades autoinmunes sistémicas

\begin{tabular}{|lc|}
\hline Lupus eritematoso sistémico & +++ \\
Artritis reumatoide & +++ \\
Síndrome Sjögren & ++ \\
Síndrome antifosfolipido & ++ \\
Dermatomiositis & ++ \\
Sarcoidosis & + \\
\hline
\end{tabular}

Nota: +++ : beneficios confirmados; ++ : beneficios probables; + : beneficios posibles. 
Está bien establecido que, independientemente de la escala de actividad que se emplee, el uso de HCQ controla la enfermedad, reduciendo la actividad, severidad y frecuencia de los brotes y disminuyendo el daño orgánico acumulado. En el mismo sentido, el tratamiento con antimaláricos mejora el perfil cardiometabólico en pacientes con LES, beneficio particularmente relevante teniendo en cuenta la aterogénesis acelerada que la enfermedad determina. En efecto, tanto en el LES como en otras EAS, se ha establecido que mejoran el perfil lipídico y el metabolismo glucídico. Se ha descripto un rol protector sobre los procesos infecciosos, el desarrollo de neoplasias, el control de la nefritis lúpica y disminución en el riesgo de trombosis ${ }^{21-23}$.

Por último, un efecto adicional, no menor, es su capacidad de comportarse como un ahorrador de corticoides. Esto también incide favorablemente en la reducción del proceso aterogénico y en la disminución de los efectos adversos -bien conocidos- de estos últimos ${ }^{24}$.

La eficacia global quedó bien establecida en un estudio pionero realizado por el "Canadian $\mathrm{Hy}$ droxychloroquine Study Group”. El estudio incluyó 47 pacientes con LES estable y bien controlado en tratamiento con HCQ. A 25 pacientes se les mantuvo el tratamiento y a 22 se les suspendió. A los 6 meses, el riesgo de brote lúpico fue 2,5 (IC 95\% 1,08-5,58) en el grupo que suspendió el tratamiento con $\mathrm{HCQ}^{25}$. El mismo grupo, en un seguimiento a 3 años, confirmó una reducción del riesgo de brotes graves del orden de $57 \%$ con el tratamiento con $\mathrm{HCQ}^{26}$. Hallazgos comparables fueron verificados en estudios posteriores ${ }^{27}$.

Los beneficios reseñados probablemente contribuyen a explicar el efecto del tratamiento con HCQ sobre la sobrevida en pacientes con LES. El primer estudio que sugirió un beneficio del tratamiento antimalárico en la sobrevida de pacientes con LES comparó pacientes fallecidos con LES respecto a no fallecidos. Los fallecidos tenían concentraciones séricas menores de cloroquina respecto a los vivos $(3,9 \mathrm{mg}$ vs $39,4 \mathrm{mg}$, $\mathrm{p}<0,001)^{28}$.

Posteriormente, Ruiz-Irastorza y cols. comprobaron, en una cohorte de 232 pacientes, que $83 \%$ de las muertes ocurrieron en pacientes nunca tratados con antimaláricos (CQ o HCQ), y 17\% en los que habían recibido antimaláricos $(\mathrm{p}<0,001)$; no observándose ninguna muerte de origen car- diovascular en pacientes que habían sido tratados con estos fármacos. La sobrevida acumulada a 15 años de los pacientes tratados con antimaláricos fue superior, con significación estadística y el HR fue de 0,14 (IC 95\% 0,04-0,48), lo que refleja una reducción promedio del riesgo de muerte de $14 \%$, con un rango que puede llegar hasta casi 50\% en los tratados con antimaláricos ${ }^{29}$. Por su parte, Alarcón y cols., en un estudio de casos y controles de la cohorte multiétnica LUMINA, compararon por cada paciente fallecido tres pacientes con vida. Luego de una mediana de seguimiento de 39 meses encontraron, en el total de la cohorte, $5 \%$ de muertes en pacientes que habían recibido HCQ versus $17 \%$ en pacientes que no habían recibido este tratamiento $(p<0,001)$. El efecto protector de HCQ sobre la sobrevida fue estadísticamente significativo, con un OR 0,319 (IC 95\% 0,118$0,864)$, lo que se traduce en una disminución del riesgo de muerte mayor a $30 \%$, comparable con el estudio anteriormente comentado ${ }^{30}$.

Por lo tanto, el tratamiento con HCQ debe iniciarse y mantenerse en todo paciente con LES, siempre que no existan contraindicaciones para ello. Debe mantenerse durante períodos de brotes graves y de remisión. Esto contradice los esquemas antiguos, en los cuales el tratamiento con HCQ era suspendido, ya sea por remisión prolongada de la enfermedad o por la asociación de otros fármacos inmunosupresores en el contexto de exacerbaciones severas $^{31}$.

\section{Hidroxicloroquina y artritis reumatoide}

Hidroxicloroquina integra el arsenal terapéutico disponible para el tratamiento de la AR y es de los denominados fármacos modificadores de la enfermedad (FAME). Produce mejoría clínica significativa y de la capacidad funcional, sin un claro efecto sobre las lesiones radiológicas (Tabla 3).

Tabla 3. Efectos del tratamiento con hidroxicloroquina en la artritis reumatoide

\begin{tabular}{|ll|}
\hline Dolor articular & Disminución \\
\hline Tumefacción articular & Disminución \\
\hline Evaluación global & Mejoría \\
\hline Riesgo cardiometabólico & Disminución \\
\hline Marcadores inflamatorios & Disminución \\
\hline
\end{tabular}


Puede ser empleado en monoterapia en pacientes con enfermedad leve. Más frecuentemente se la utiliza como coadyuvante con otros FAME, como metotrexate (MTX), leflunomida (LFN) o sulfasalazina $^{32}$. Además, recientemente se han descrito beneficios de este medicamento vinculados a su acción sobre el metabolismo lipídico y la insulinoresistencia en pacientes con AR, semejante a lo descripto en el LES ${ }^{33}$.

Por otro lado, el uso de HCQ en combinación con otros FAME está bien documentado en la literatura. Varios estudios apoyan un tratamiento intensivo y temprano de la AR, en tanto el control rápido y mantenido de la enfermedad permite enlentecer el desarrollo de las lesiones radiológicas $^{34}$. Es necesario tener en cuenta que el tratamiento con HCQ no retrasa la progresión del daño articular en la misma medida que otros FAME, pero su utilidad radica en que en el caso de AR con niveles de actividad bajos, la lesión articular también es baja ${ }^{35}$.

Las principales sociedades científicas internacionales recomiendan el uso de HCQ como FAME inicial en pacientes con síntomas leves de AR y sin factores de mal pronóstico. Además, debe tenerse en cuenta la posibilidad de emplear HCQ junto a otros FAME ${ }^{35}$.

\section{Hidroxicloroquina en el síndrome de Sjögren}

Desde hace varios años se emplea HCQ en el tratamiento del síndrome de Sjögren (SS). Algunos estudios han sugerido que mejora la actividad inflamatoria a nivel glandular, los síntomas secos que caracterizan a la enfermedad (xerostomía y xeroftalmia), otros síntomas extraglandulares como artralgias y fatiga, además de mejorar los marcadores inflamatorios sistémicos como la VSE, la proteína $C$ reactiva (PCR) y la hipergammaglobulinemia (Tabla 4). El mecanismo por el cual determina estos efectos no está completamente

Tabla 4. Efectos del tratamiento con hidroxicloroquina en el síndrome de Sjögren

\begin{tabular}{|ll|}
\hline Marcadores inflamatorios sistémicos & Disminución \\
\hline Calidad lacrimal & Mejoría \\
Xerooftalmia & Mejoría \\
Xerostomía & Mejoría \\
\hline
\end{tabular}

aclarado, pero se ha sugerido que inhibe la actividad de la colinesterasa a nivel glandular, lo cual mejoraría la secreción de las glándulas salivales y lacrimales ${ }^{36}$.

Un estudio, que incluyó 32 pacientes de sexo femenino que venían siendo tratadas con HCQ por al menos 2 años, a dosis de $6,5 \mathrm{mg} / \mathrm{kg} /$ día y que evaluó el efecto de suspender el tratamiento durante 12 semanas, mostró que quienes suspendían el tratamiento empeoraban en parámetros subjetivos de sequedad ocular. No se constataron diferencias en el test de Schirmer, pero sí en los test cualitativos de estabilidad de la película lacrimal. Estos últimos empeoraron al suspender el tratamiento con HCQ, lo que apoyaría su uso en el síndrome seco ${ }^{37}$. Un estudio retrospectivo, que incluyó 35 pacientes con síndrome seco que recibieron HCQ por 5 meses, observó mejoría en parámetros objetivos de secreción glandular salival y lacrimal. Los pacientes que presentaban anticuerpos $\mu$-fodrina fueron los que presentaron mejor respuesta, lo que sugiere que los que tienen mayor actividad inflamatoria tendrán una mejor respuesta al tratamiento con $\mathrm{HCQ}^{38}$. Por tanto, si bien los estudios tienen sesgos y limitaciones, sugieren que el tratamiento con HCQ determina un beneficio principalmente en los síntomas glandulares de esta enfermedad ${ }^{39}$.

\section{Hidroxicloroquina en el síndrome antifosfolípido}

Ya se ha mencionado el efecto antitrombótico de HCQ. En la década de 1980-89 se utilizó como profilaxis de la enfermedad tromboembólica venosa en pacientes sometidos a prótesis de cadera ${ }^{40}$. El tratamiento con HCQ también ha demostrado beneficios en el síndrome antifosfolípido (SAF) y en potadores de anticuerpos antifosfolípidos (AAF). Se ha descrito que disminuye la agregación plaquetaria, la viscosidad sanguínea y la aglutinación de los glóbulos rojos en sangre periférica. Esto fue inicialmente probado en un estudio con ratones, a los que se les inyectó anticuerpos antifosfolípidos y luego se les provocó una lesión vascular. Los que recibieron HCQ desarrollaron un trombo más pequeño y de menor duración respecto a los no tratados con este medicamento ${ }^{41}$.

Se ha evidenciado que HCQ determina una disociación de la unión de los anticuerpos antib2-glicoproteína-1 (anti-Gp1) de tipo IgG a la bicapa 
fosfolipídica. También se ha demostrado que HCQ reduce la activación plaquetaria mediada por $\mathrm{AAF}$, la agregación plaquetaria inducida por colágeno, la liberación de los gránulos plaquetarios y la expresión de glicoproteína IIb-IIIa plaquetaria inducida por los $\mathrm{AAF}^{42}$. Los AAF también interfieren con la función de la anexina A5, potente proteína anticoagulante vascular y placentaria. Estudios experimentales han confirmado que HCQ reduce la unión de los AAF a la anexina A5, permitiendo restaurar la actividad esta última ${ }^{43}$. Por lo tanto, estos efectos actuarían previo a la formación del trombo y podrían jugar un rol importante en la prevención del desarrollo de los eventos trombóticos del SAF. Por otro lado, se ha descrito la capacidad de este fármaco de reducir los títulos de AAF seguidos a largo plazo en pacientes con LES y SAF ${ }^{44}$. A estos efectos antitrombóticos se agregan sus efectos inmumoduladores descritos previamente, por lo que la vía final del efecto antitrombótico de HCQ es producto de varias vías de acción.

Si bien es cierto que los beneficios del uso de HCQ en el SAF han sido comprobados fundamentalmente en pacientes con LES, también existe evidencia que sugiere que su uso es protector en pacientes con SAF sin LES o en portadores de $\mathrm{AAF}^{45}$. Es bien conocido que, a pesar de los avances en el tratamiento antitrombótico, la recurrencia de los eventos trombóticos en pacientes con SAF $-10 \%$ al año del primer evento y $20 \%$ a los 5 añossigue siendo motivo de preocupación ${ }^{46}$.

Existe evidencia creciente del uso de HCQ en pacientes con SAF refractario a tratamientos convencionales o recurrentes bajo medicación antitrombótica. Un estudio clínico prospectivo no aleatorizado, desarrollado por un grupo francés, incluyó 40 pacientes con SAF con eventos tromboembólicos venosos, no arteriales ni obstétricos. Veinte pacientes fueron tratados con dicumarínicos con INR objetivo entre 2 y 3 y otros 20 con el mismo tratamiento más HCQ. En el segundo grupo no hubo recurrencia de eventos tromboembólicos comparado con 30\% de recurrencia en el grupo que sólo recibió dicumarínicos a tres años de seguimiento. No hubo diferencias en la tasa de complicaciones hemorrágicas entre ambos grupos. Por lo tanto, el tratamiento con HCQ podría llegar a constituir una herramienta valiosa en la prevención secundaria del SAF primario ${ }^{47}$.

En relación al uso de HCQ en pacientes por- tadores de AAF sin eventos tromoboembólicos ni obstétricos ni otras EAS, se está desarrollando un ensayo clínico multinacional con el propósito de determinar su efecto en prevención primaria. Se conoce que en pacientes con LES portadores de AAF el uso de dosis bajas de ácido acetil salicílico más HCQ reduce la frecuencia de complicaciones tromboembólicas ${ }^{48}$.

\section{Hidroxicloroquina en otras enfermedades autoinmunes sistémicas}

Dermatomiositis y polimiositis constituyen dos miopatías inflamatorias idiopáticas. $\mathrm{Al}$ igual que ocurre con otras EAS, la base del tratamiento lo constituye el uso de glucocorticoides en conjunto con inmunosupresores. Los ensayos clínicos son escasos en estas enfermedades, por lo que la información procede de series de casos, estudios retrospectivos u opinión de expertos. Además, no en todos los trabajos se emplean los mismos criterios clasificatorios de estas entidades nosológicas, lo que dificulta aún más extraer conclusiones válidas y extrapolables a la práctica clínica. Sin embargo, varios trabajos han sugerido que el uso de HCQ determina beneficios en el manejo de las manifestaciones cutáneas pero no de las musculares. Este efecto podría deberse, además de a su efecto inmunomodulador, a la capacidad de HCQ de proteger de la radiación ultravioleta, lo que parece particularmente relevante en lesiones fotosensibles. Algunos estudios han evidenciado una mejora de hasta $75 \%$ de las lesiones cutáneas. Si bien se requiere un mayor número de trabajos para confirmar los beneficios del uso de HCQ en esta enfermedad, parece razonable su empleo, particularmente en lesiones cutáneas ${ }^{49}$.

En sarcoidosis también existen reportes que sugieren beneficios del tratamiento con HCQ, particularmente en lesiones musculoesqueléticas, ganglionares y pulmonares ${ }^{50}$. Algo similar ocurre en la enfermedad de Still, en que se han sugerido beneficios del tratamiento con HCQ en combinación con metotrexate y sulfasalazina ${ }^{51}$.

\section{Conclusiones}

La eficacia del tratamiento con HCQ ha sido probada en el tratamiento del LES y de la AR. Se 
destaca su impacto favorable en diversas variables clínicas y particularmente en el aumento de la sobrevida en el LES. En el síndrome de Sjögren su eficacia es de menor impacto, pero igualmente importante en una enfermedad con opciones terapéuticas limitadas. En el síndrome antifosfolipídico se destaca que su efecto antitrombótico es especialmente atractivo como terapia adyuvante en pacientes con eventos trombóticos recurrentes. $\mathrm{Su}$ efecto en prevención primaria en pacientes portadores de AAF sin LES está siendo estudiado y aún no está claramente establecido.

En otras entidades como dermatomiositis y polimiositis los datos son menos contundentes pero igualmente relevantes respecto a beneficios en manifestaciones cutáneas. Algo similar ocurre en el tratamiento de la sarcoidosis. En la enfermedad de Still podría jugar un rol en pacientes que ya están tratados y que no han respondido a tratamientos convencionales.

$\mathrm{Su}$ perfil de seguridad es muy favorable. Sus efectos adversos son escasos. La toxicidad retiniana es motivo de preocupación aunque su frecuencia no es elevada. Se requieren controles periódicos para pesquisar precozmente lesiones predisponentes y potencialmente reversibles. Otros efectos adversos deben ser tenidos en cuenta y monitorizados. Puede ser usada con razonable seguridad en el embarazo y la lactancia.

\section{Mensajes clave}

Hidroxicloroquina controla la actividad inflamatoria modulando al sistema inmune por varios mecanismos.

Tiene otros efectos beneficiosos: antitrombótico, hipolipemiante, hipoglicemiante y disminuye el riesgo de malignidad.

Su eficacia ha sido comprobada específicamente en el LES y la AR.

En el LES ha demostrado aumentar la sobrevida.

Su perfil de seguridad es muy favorable.

Si bien su uso requiere control oftalmológico las complicaciones más preocupantes son poco frecuentes.

\section{Referencias}

1. Pallarés Ferreres L, Julià Benique MR, Esteban Marcos E, Rascón Rico J. Orientación diagnóstica basada en la clínica y el laboratorio. En: Ramos Casals M, García
Carrasco M, Gómez de Salazar JR, Calvo Alén J, Font Franco J, Editores, Enfermedades Autoinmunes Sistémicas y Reumatológicas. Barcelona, España: Editorial Masson S.A.; 2005. p. 45-59.

2. Lisnevskaia L, Murphy G, Isenberg D. Systemic lupus erythematosus. Lancet 2014; 384 (9957): 1878-88.

3. Smolen JS, Aletaha D. Rheumatoid arthritis therapy reappraisal: strategies, opportunities and challenges. Nat Rev Rheumatol 2015. doi: 10.1038/nrrheum.2015.8. [Epub ahead of print].

4. Wallace DJ. The use of quinacrine (Atabrine) in rheumatic diseases: a reexamination. Semin Arthritis Rheum 1989; 18 (4): 282-96.

5. Furst DE. Pharmacokinetics of hydroxychloroquine and chloroquine during treatment of rheumatic diseases. Lupus 1996; 5 Suppl 1: S11.

6. Rainsford KD, Parke AL, Clifford-Rashotte M, Kean WF. Therapy and pharmacological properties of hydroxychloroquine and chloroquine in treatment of systemic lupus erythematosus, rheumatoid arthritis and related diseases. Inflammopharmacology. 2015 Aug 6 [Epub ahead of print].

7. Leroux G, Costedoat-Chalumeau N, Hulot J-S, et al. Relationship between blood hydroxychloroquine concentrations and cigarette smoking in trated patients with connective tissue diseases. Ann Rheum Dis 2007; 66 (11): 1547-8.

8. Chasset F, Frances C, Barete S. Influence of smoking on the efficacy of antimalarials in cutaneous lupus: a meta-analysis of the literatura. J Am Acad Dermatol 2015; 72 (4): 634-9.

9. Costedoat-Chalumeau N, Dunogué B, Morel N, Le Guern V, Guettrot-Imbert G. Hydroxychloroquine: a multifaceted treatment in lupus. Presse Med 2014; 43: 167-80.

10. Wallace DJ, Gudsoorkar VS, Weisman MH, Venuturupalli SR. New insights into mechanisms of therapeutic effects of antimalarial agents in SLE. Nat Rev Rheumatol 2012; 8 (9): 522-33.

11. Olsen NJ, Schleich MA, Karp DR. Multifaceted effects of hydroxychloroquine in human disease. Semin Arthritis Rheum 2013; 43: 264-72.

12. Kalia S, Dutz JP. New concepts in antimalarial use and mode of action in dermatology. Dermatol Ther 2007; 20: 160-74.

13. Herman SM, Shin MH, Holbrook A, Rosenthal D. The role of antimalarials in the exacerbation of psoriasis: a systematic review. Am J Clin Dermatol 2006; 7: 24957.

14. Stelton CR, Connors DB, Walia SS, Walia HS. Hydrochloroquine retinopathy: characteristic presentation 
with review of screening. Clin Rheumatol 2013; 32: 895-8.

15. Ruiz-Irastorza G, Ramos-Casals M, Brito-Zeron P, Khamashta MA. Clinical efficacy and side effects of antimalarials in systemic lupus erythematosus: a systematic review. Ann Rheum Dis 2010; 69: 20-8.

16. Marmor MF, Kellner U, Lai TY, Lyons JS, Mieler WF. Revised recommendations on screening for chloroquine and hydroxychloroquine retinopathy. Ophthalmology 2011; 118: 415-22.

17. Costedoat-Chalumeau N, Hulot JS, Amoura Z, Delcourt A, Maisonobe T, Dorent R, et al. Cardiomyopathy related to antimalarial the-rapy with Iillustrative case report. Cardiology 2006; 107: 73-80.

18. Østensen M, Andreoli L, Brucato A, Cetin I, Chambers C, Clowse ME, et al. State of the art: Reproduction and pregnancy in rheumatic diseases. Autoimmun Rev 2015; 14 (5): 376-86.

19. Danza A, Díaz L, Ruiz-Irastorza G. Hidroxicloroquina en el embarazo y la lactancia. Rev Med Urug 2015; 31 (2): 128-32.

20. Ruiz-Irastorza G, Khamashta MA. Hydroxychloroquine: the cornerstone of lupus therapy. Lupus 2008; 17: 271-3.

21. Fessler BJ, Alarcón GS, McGwin G Jr, Roseman J, Bastian HM, Friedman AW, et al. LUMINA Study Group. Systemic lupus erythematosus in three ethnic groups: XVI. Association of hydroxychloroquine use with reduced risk of damage accrual. Arthritis Rheum 2005; 52 (5): 1473-80.

22. Petri M. Hydroxychloroquine use in the Baltimore Lupus Cohort: effects on lipids, glucose and thrombosis. Lupus 1996; 5 Suppl 1: S16-22.

23. Kasitanon N, Fine DM, Haas M, Magder LS, Petri M. Hydroxychloroquine use predicts complete renal remission within 12 months among patients treated with mycophenolate mofetil therapy for membranous lupus nephritis. Lupus 2006; 15 (6): 366-70.

24. Ruiz-Irastorza G, Danza A, Khamashta M. Glucocorticoid use and abuse in SLE. Rheumatology (Oxford) 2012; 51 (7): 1145-53.

25. A randomized study of the effect of withdrawing hydroxychloroquine sulfate in systemic lupus erythematosus. The Canadian Hydroxychloroquine Study Group. N Engl J Med 1991; 324 (3): 150-4.

26. Tsakonas E, Joseph L, Esdaile JM, Choquette D, Senécal JL, Cividino A, et al. A long-term study of hydroxychloroquine withdrawal on exacerbations in systemic lupus erythematosus. The Canadian Hydroxychloroquine Study Group. Lupus 1998; 7 (2): 80-5.

27. Akhavan PS, Su J, Lou W, Gladman DD, Urowitz MB, Fortin PR. The early protective effect of hydroxychloro- quine on the risk of cumulative damage in patients with systemic lupus erythematosus. J Rheumatol 2013; 40 (6): 831-41.

28. Hernández-Cruz B, Tapia N, Villa-Romero AR, Reyes E, Cardiel MH. Risk factors associated with mortality in systemic lupus erythematosus. A case-control study in a tertiary care center in Mexico City. Clin Exp Rheumatol 2001; 19 (4): 395-401.

29. Ruiz-Irastorza G, Egurbide MV, Pijoan JI, Garmendia M, Villar I, Martínez-Berriotxoa A, et al. Effect of antimalarials on thrombosis and survival in patients with systemic lupus erythematosus. Lupus 2006; 15 (9): 577 83.

30. Alarcón GS, McGwin G, Bertoli AM, Fessler BJ, Calvo-Alén J, Bastian HM, et al. LUMINA Study Group. Effect of hydroxychloroquine on the survival of patients with systemic lupus erythematosus: data from LUMINA, a multiethnic US cohort (LUMINA L). Ann Rheum Dis 2007; 66 (9): 1168.

31. Ruiz-Irastorza G, Danza A, Khamashta M. Tratamiento del lupus eritematoso sistémico: mitos, certezas y dudas. Med Clin (Barc) 2013; 141 (12): 533-42.

32. Gaujoux-Viala C, Smolen JS, Landewé R, Dougados M, Kvien TK, Mola EM, et al. Current evidence for the management of rheumatoid arthritis with synthetic disease-modifying antirheumatic drugs: a systematic literature review informing the EULAR recommendations for the management of rheumatoid arthritis. Ann Rheum Dis 2010; 69 (6): 1004-9.

33. Bili A, Sartorius JA, Kirchner HL, Morris SJ, Ledwich LJ, Antohe JL, et al. Hydroxychloroquine use and decreased risk of diabetes in rheumatoid arthritis patients. J Clin Rheumatol 2011; 17 (3): 115-20.

34. Saunders SA, Capell HA, Stirling A, Vallance R, Kincaid $\mathrm{W}$, McMahon AD, et al. Triple therapy in early active rheumatoid arthritis: a randomized, single-blind, controlled trial comparing step-up and parallel treatment strategies. Arthritis Rheum 2008; 58 (5): 1310-7.

35. Smolen JS, Landewé R, Breedveld FC, Buch M, Burmester $\mathrm{G}$, Dougados $\mathrm{M}$, et al. EULAR recommendations for the management of rheumatoid arthritis with synthetic and biological disease-modifying antirheumatic drugs: 2013 update. Ann Rheum Dis 2014; 73 (3): 492-509.

36. Brito-Zerón P, Sisó-Almirall A, Bové A, Kostov BA, Ramos-Casals M. Primary Sjögren syndrome: an update on current pharmacotherapy options and future directions. Expert Opin Pharmacother 2013; 14 (3): 279-89.

37. Yavuz S, Asfuro lu E, Bicakcigil, Toker E. Hydroxychloroquine improves dry eye symptoms of patients with primary Sjogren's syndrome. Rheumatol Int 2011; 31 (8): 1045-9. 
38. Rihl M, Ulbricht K, Schmidt RE, Witte T. Treatment of sicca symptoms with hydroxychloroquine in patients with Sjogren's syndrome. Rheumatology (Oxford) 2009; 48 (7): 796-9.

39. Ramos-Casals M, Tzioufas AG, Stone JH, Sisó A, Bosch $\mathrm{X}$. Treatment of primary Sjögren syndrome: a systematic review. JAMA 2010; 304 (4): 452-60.

40. Loudon JR. Hydroxychloroquine and postoperative thromboembolism after total hip replacement. Am J Med 1988; 85: 57-61.

41. Edwards MH, Pierangeli S, Liu X, Barker JH, Anderson G, Harris EN. Hydroxychloroquine reverses thrombogenic properties of antiphospholipid antibodies in mice. Circulation 1997; 96: 4380-4.

42. Rand JH, Wu XX, Quinn AS, Chen PP, Hathcock JJ, Taatjes DJ. Hydroxychloroquine directly reduces the binding of antiphospholipid antibody-beta2-glycoprotein I complexes to phospholipid bilayers. Blood 2008; 112 (5): 1687-95.

43. Rand JH, Wu XX, Quinn AS, Ashton AW, Chen PP, Hathcock JJ, et al. Hydroxychloroquine protects the annexin A5 anticoagulant shield from disruption by antiphospholipid antibodies: evidence for a novel effect for an old antimalarial drug. Blood 2010; 115 (11): 2292-9.

44. Petri M. Use of Hydroxychloroquine to prevent thrombosis in systemic lupus erythematosus and antiphospholipid antibody-positive patients. Curr Rheumatol Rep 2011; 13: 77-80.

45. Belizna C. Hydroxychloroquine as anti-thrombotic in antiphospholipid syndrome. Autoimmun Rev 2015; 14: 358-62.

46. Cervera R, Khamashta MA, Shoenfeld Y, Camps MT, Jacobsen S, Kiss E, et al. Euro-Phospholipid Project Group (European Forum on Antiphospholipid Antibodies). Morbidity and mortality in the antiphospholipid syndrome during a 5-year period: a multicentre prospective study of 1000 patients. Ann Rheum Dis 2009; 68 (9): 1428-32.

47. Schmidt-Tanguy A, Voswinkel J, Henrion D, Subra JF, Loufrani L, Rohmer V, et al. Antithrombotic effects of hydroxychloroquine in primary antiphospholipid syndrome patients. J Thromb Haemost 2013; 11 (10): 1927-9.

48. Broder A, Putterman C. Hydroxychloroquine use is associated with lower odds of persistently positive antiphospholipid antibodies and/or lupus anticoagulant in systemic lupus erythematosus. J Rheumatol 2013; 40 (1): 30-3.

49. Femia AN, Vleugels RA, Callen JP. Cutaneous dermatomyositis: an updated review of treatment options and internal associations. Am J Clin Dermatol 2013; 14 (4): 291-313.

50. Baughman RP, Lower EE. Medical therapy of sarcoidosis. Semin Respir Crit Care Med 2014; 35 (3): 391-406.

51. Carreño MA, Carvallo A, Trejo C, Ballestero F, Martínez C. Adult Still's disease. A great simulator. Retrospective review of 20 patients. Rev Med Chile 2009; 137 (8): 1010-6. 